\title{
Migration de prothèse herniaire en intravésical : mécanisme et enjeux
}

\author{
Jihad El anzaoui, MD ; Omar Ghoundale, MD ; ${ }^{*}$ Driss Touiti, MD ; ${ }^{*}$ Pierre Henri Savoie, MD ; \\ Julien Laroche, MD ; Philipe Riviere, MD ;† Loic Lemesle, MD ; Robert Fournier, MD ${ }^{\dagger}$
}

*Service d'urologie, Hôpital militaire Avicenne, Marrakech, Maroc; 'Service d'urologie, Hôpital Sainte Anne, Toulon, France

Cite as: Can Urol Assoc J 2015;9(7-8):E458-62. http://dx.doi.org/10.5489/cuai.2640 Published online July 17, 2015.

\section{Résumé}

La migration d'une prothèse synthétique pour hernie abdominale ou inguinale est un incident rare. Elle peut survenir aussi bien en intrapéritonéal qu'en sous-péritonéal. La migration intrapéritonéale entraîne un risque d'obstruction intestinale et d'infection pouvant menacer le pronostic vital. La migration endovésicale est beaucoup plus rare. La plaque en endovésical se comporte comme un corps étranger avec risque de calcification et de cystite. Le diagnostic différentiel se pose surtout avec un calcul vésical ou une tumeur de la vessie. Devant la prédominance de signes irritatifs non spécifiques, le diagnostic repose sur les observations radiologiques mais surtout cystoscopiques. Le traitement est essentiellement endoscopique, par la résection de l'extrémité prolabée en endovésical.

\section{Introduction}

Grâce à sa simplicité et son efficacité, la prothèse synthétique est devenue le traitement de référence en matière de chirurgie herniaire. La migration de la prothèse est une complication tardive mais grave qui peut menacer le pronostic vital. La migration peut être intrapéritonéale ou sous-péritonéale. La migration intravésicale est un incident rare ${ }^{1}$, mais étant donné la fréquence de pose de prothèses herniaires dans le monde, certains auteurs n'écartent pas le fait qu'il $s^{\prime}$ agit d'un phénomène plus fréquent mais sous-diagnostiqué et peu étudié ${ }^{2}$.

\section{Hernioplastie et biomatériels}

Lischtenstein fut le premier à introduire le procédé sans tension dans la chirurgie visant à corriger I'hernie inguinale ${ }^{3}$. Ce procédé permet, par la pose d'une prothèse, de renforcer les structures musculofasciales de la région inguinale sans pour autant pratiquer une large dissection ni des plicatures forcées pouvant conduire à des déchirures tissulaires.

Après une exposition du sac herniaire et son refoulement, la technique consiste à poser une plaque de tissu synthétique, fixée par du fil non résorbable, aux structures ligamento-aponévrotiques de la région inguinale, permettant de renforcer le fascia transversalis et de cravater sans stranguler le cordon spermatique au niveau du canal inguinal profond.

Le rôle de la prothèse n'est pas de remplacer la paroi abdominale mais plutôt de la renforcer par la création d'une réaction inflammatoire locale qui stimule l'activité fibroblastique. Cette technique attrayante par sa simplicité et son efficacité fut étendue à toute la chirurgie herniaire, notamment la cure d'éventration abdominale. Le choix d'un type de prothèse demeure une difficulté pour tout chirurgien viscéraliste. Différents types de prothèses existent, qui diffèrent principalement par leur caractère biologique ou synthétique, résorbable ou non résorbable.

Depuis 1994, année où Amid et al. ont publié une classification des prothèses non résorbables ${ }^{4}$, différentes classifications se sont succédé devant l'expansion importante de I'industrie prothétique ${ }^{5}$.

Malheureusement, aucun type de prothèse ne possède les caractéristiques $d^{\prime}$ 'une prothèse idéale et les complications liées aux prothèses continuent toujours d'alimenter la littérature. Les mécanismes derrière les complications peuvent être variés $^{6}$. Nous proposons dans le tableau 1 une description des différentes complications pouvant être rencontrées au cours de l'insertion d'une prothèse herniaire.

\section{Migration de la prothèse herniaire}

La migration de prothèse est une séparation suivie d'un déplacement de la prothèse en dehors de son site de pose. Cette complication reste rare et peu rapportée dans la littérature. 


\section{Mécanisme}

La physiopathologie de la migration reste mal connue. Ce n'est que récemment que ce problème a suscité l'intérêt des auteurs. En 2006, Agrawal fut le premier à tenter d'expliquer le mécanisme de migration prothétique, suggérant deux mécanismes ${ }^{7}$.

Le premier est un mécanisme dit primaire selon lequel une prothèse peut migrer suivant les plans de faible résistance tissulaire. Ceci peut être dû selon l'auteur à une mauvaise fixation de la prothèse. Le deuxième est un mécanisme dit secondaire où la prothèse, par une réaction à corps étranger, fragilise et érode les tissus, ce qui favorise sa migration.

Ces deux mécanismes font ressortir l'importance de deux facteurs, soit la qualité de la fixation et la nature physicochimique de la plaque. En effet, un grand débat est ouvert depuis quelques années sur la nécessité de fixer la plaque de cure de l'hernie ${ }^{8}$. Selon certains auteurs, ne pas fixer la plaque permet de gagner du temps, évite les lésions nerveuses, minimise l'inflammation et la douleur postopératoire, et diminue les coûts sans pour autant, à ce qu'il paraît, augmenter le risque de récidives ou de complications. Ainsi, des prothèses à fixation spontanée ont vu le jour (figure 1).

Le lien théorique entre la non-fixation de la plaque et le risque de migration n'a pas encore été mis en évidence en pratique. En ce qui concerne le rapport entre la nature physicochimique de la plaque et sa migration, il existe une multitude de types de prothèses sur le marché (tableau 2) différant selon le poids, la largeur de leurs mailles, la résistance à l'infection et le coût. Toutes ces plaques entraînent une réaction à corps étranger dont l'intensité diffère d'une plaque à l'autre ${ }^{9}$.

Cette réaction, selon son intensité, fragilise les tissus avoisinants et peut être impliquée dans la migration de la prothèse et l'érosion des structures avoisinantes. En contrepartie, cette réaction inflammatoire aide à l'incorporation de la plaque au site d'implantation ${ }^{10}$. Mais aucune étude n'a essayé de clarifier cette question.

La prothèse idéale reste à déterminer. Elle est définie comme étant celle qui comporte à la fois une faible réaction inflammatoire mais aussi une intense activité fibroblastique.

\begin{tabular}{|c|c|}
\hline Mécanisme & Définition \\
\hline Contraction & Diminution de la surface de la prothèse \\
\hline Séparation & Déconnexion du site d'ancrage \\
\hline Exposition & Accessible à la palpation ou à l'inspection visuelle \\
\hline Extrusion & $\begin{array}{l}\text { Passage dans un organe creux (p. ex. intestin, } \\
\text { vessie, vagin) ou à travers la peau }\end{array}$ \\
\hline Infection & $\begin{array}{l}\text { Formation d'abcès et de fistules. Réaction } \\
\text { inflammatoire systémique. }\end{array}$ \\
\hline Déplacement & Situation en dehors du site de pose \\
\hline
\end{tabular}

\begin{tabular}{lcc}
\hline $\begin{array}{l}\text { Tableau } 2 \text { : Classification d'Amid pour les prothèses non } \\
\text { résorbables }\end{array}$ & Exemple \\
\hline Type & Caractéristiques & Marlex (CR Bard) \\
& & Prolene (Ethicon) \\
& & Atrium (Atrium) \\
& & Prolite (Atrium) \\
& & Trelex (Meadox) \\
Type I & Macroporeuse $(>75 \mu \mathrm{m})$ & Atrium (Atrium) \\
& monofilament & Premilene (B-Braun) \\
& & Serapren (smooth) \\
& & Parietene (Covidien) \\
& & Gore Tex (WL Gore) \\
Type II & Microporeuse (<10 $\mu \mathrm{m})$ & \\
& Macroporeuse & Mersilene (Ethicon) \\
Type III & multifilament ou associée & Teflon (CR Bard) \\
& à une composante & \\
Type IV & microporeuse & Silastic \\
\hline
\end{tabular}

Des migrations de plaque ont été observées avec tous les types de plaques. En plus des deux mécanismes évoqués par Agrawal, d'autres facteurs peuvent être soupçonnés :

- Lésion viscérale par les bords aigus d'une prothèse mal taillée ${ }^{11}$; ce risque est spécialement majoré lors du traitement d'éventration abdominale par la pose de plaques en prépéritonéal ou intrapéritonéal. Les prothèses étendues en sous-ombilical peuvent être directement mises en contact avec la paroi vésicale, d'où un risque de blessure de cette paroi, notamment en cas d'hernie vésicale (figure 2, figure 3).

- Coinçage par les fils de suture.

- Lésion d'un organe au cours de l'hernioplastie. Ce risque est plus majoré au cours de l'hernioplastie inguinale. Les points de fixation de la prothèse au tendon conjoint comportent le risque de la prise de la paroi latérale $d^{\prime}$ une vessie pleine.

- L'infection de la plaque peut aussi être un facteur causal de la migration et de fistules.

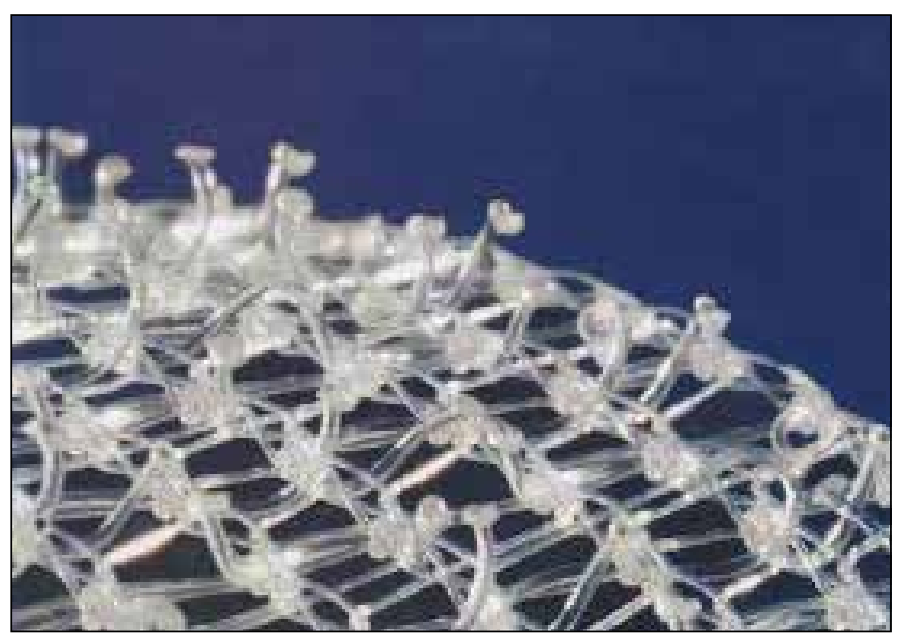

Fig. 1. Plaque Progrip à fixation spontanée. 


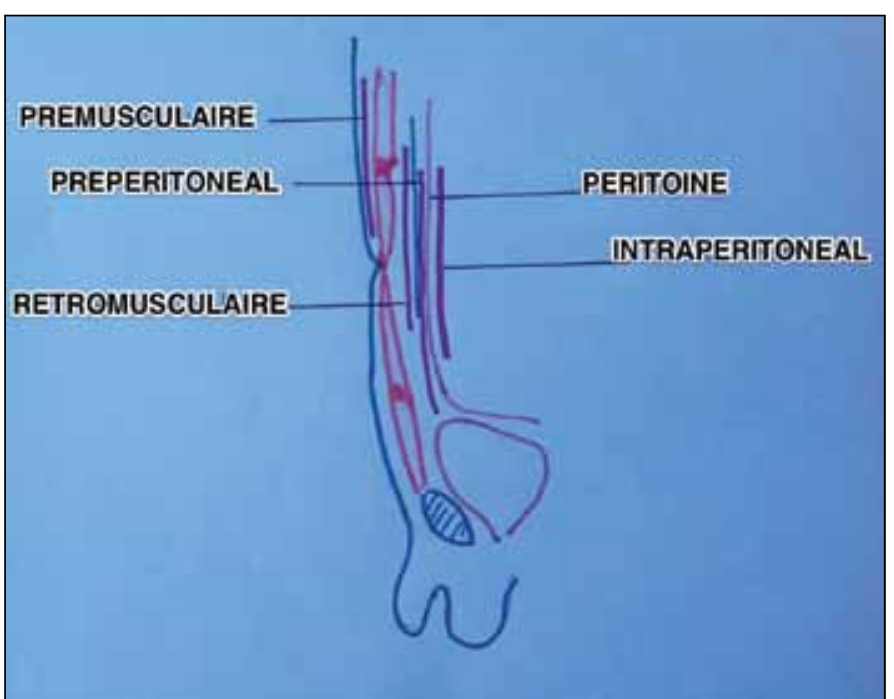

Fig. 2. Coupe sagittale montrant les différents sites de pose d'une prothèse dans le traitement d'éventration abdominale.

- Une fois séparée de son site de fixation, la plaque peut glisser en direction des organes intrapéritonéaux ou en sous-péritonéal.

\section{Migration intrapéritonéale}

La migration intrapéritonéale est plus connue et plus fréquemment rapportée dans la littérature. Des cas d'extrusion de prothèse dans le colon, dans l'intestin grêle ou dans les annexes ont été rapportés ${ }^{12-14}$.

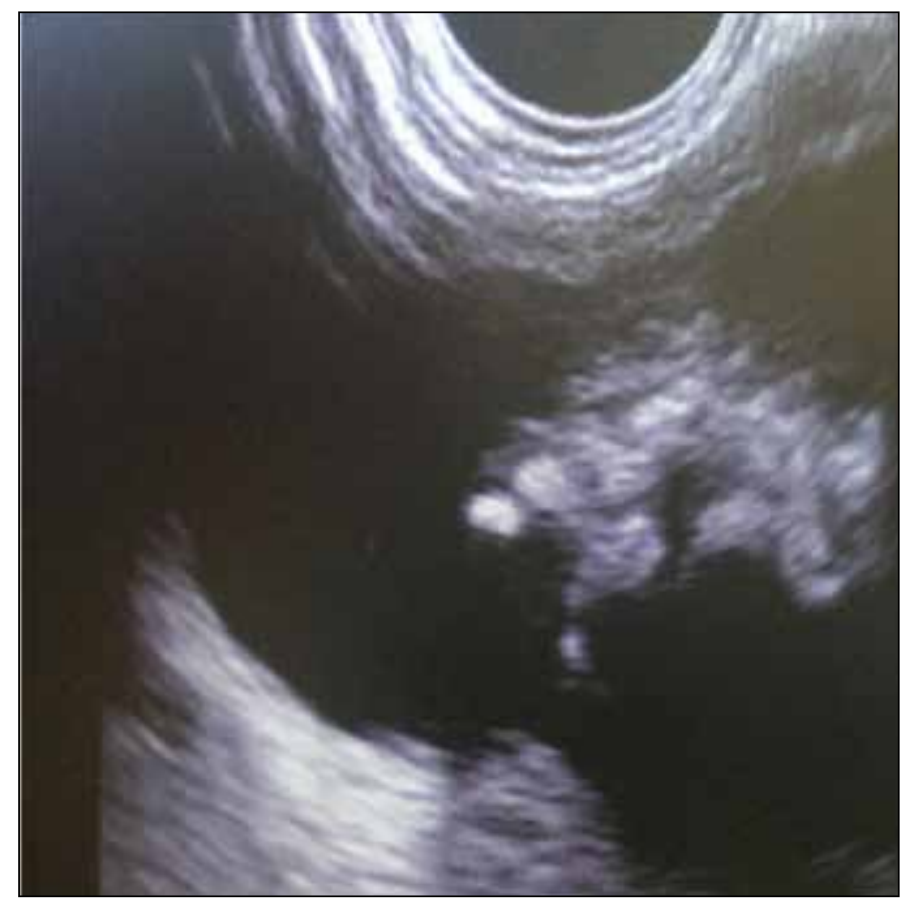

Fig. 4. Image échographique : structure hyperéchogène dans la lumière vésicale avec un cône d'ombre postérieur évoquant un calcul vésical.

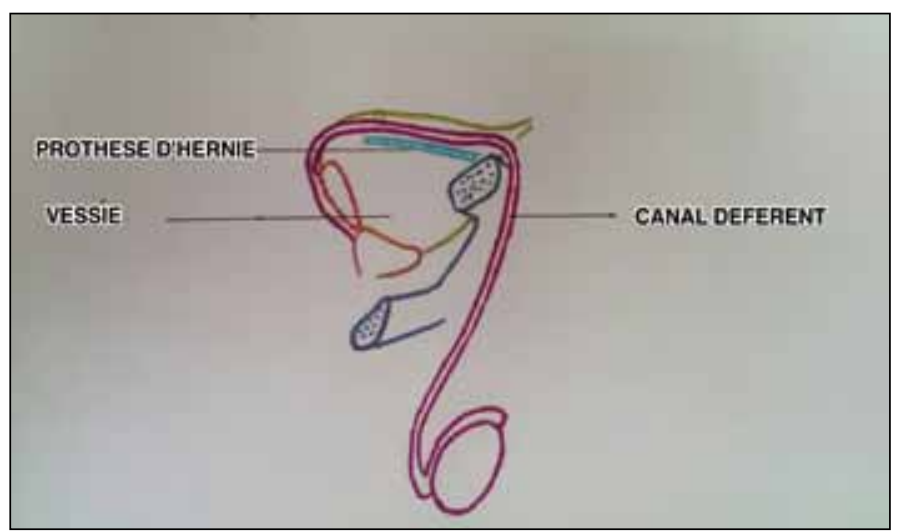

Fig. 3. Coupe parasagittale passant par le canal inguinal superficiel montrant le plan de pose d'une prothèse au cours d'une hernioplastie inguinale.

La gravité da la migration intrapéritonéale réside dans le risque d'érosion des structures intra-abdominales, la formation d'abcès et de fistules extériorisées ou non.

La symptomatologie diffère de la simple douleur à l'occlusion et la péritonite, en passant par le saignement digestif et le sepsis ${ }^{15}$. La récidive de l'hernie n'est pas systématique.

Le diagnostic radiologique n'est pas toujours aisé. La TDM permet une meilleure exploration de la cavité péritonéale que l'échographie. Elle constitue l'examen de référence pour cette indication ${ }^{16}$, permettant $\mathrm{d}^{\prime}$ objectiver la prothèse migrée, le type de migration et ses conséquences comme I'occlusion, les abcès et les trajets fistuleux.

\section{Migration intravésicale}

La migration intravésicale est une séparation suivie $d^{\prime}$ un déplacement puis d'une extrusion de la plaque dans la vessie. Le délai entre l'hernioplastie et la migration de la plaque est variable, allant de quelques mois à plusieurs années. Des facteurs de risque ont été mentionnés dans la littérature : I'abord laparoscopique, l'absence de drainage postopératoire, la plénitude vésicale au cours de l'hernioplastie ${ }^{17,18}$.

Le diagnostic n'est pas souvent facile à cause de la réaction à corps étranger induite par l'irritation causée par la prothèse. Le bout de prothèse intravésicale peut se calcifier, faisant errer le diagnostic vers un calcull ${ }^{15}$, ou induire une hypertrophie muqueuse imitant une tumeur vésicale ${ }^{19}$. Le diagnostic dans ces cas est redressé en peropératoire. Cliniquement, la symptomatologie irritative est dominante et peut être associée ou non à une hématurie.

L'hématurie isolée est un signe clinique très fréquent ${ }^{1,2}$.

On peut aussi observer des infections urinaires à répétition $^{20}$, une masse vésicale ${ }^{2}$ ou un abcès de la paroi abdominale ${ }^{18}$. La plaque reste difficile à objectiver par l'échographie ou la TDM avec produit de contraste. L'aspect typique est une protrusion de la prothèse herniaire à travers un point faible de la paroi vésicale. Des images de calcification ou de végétations sont beaucoup plus fréquentes et peuvent 


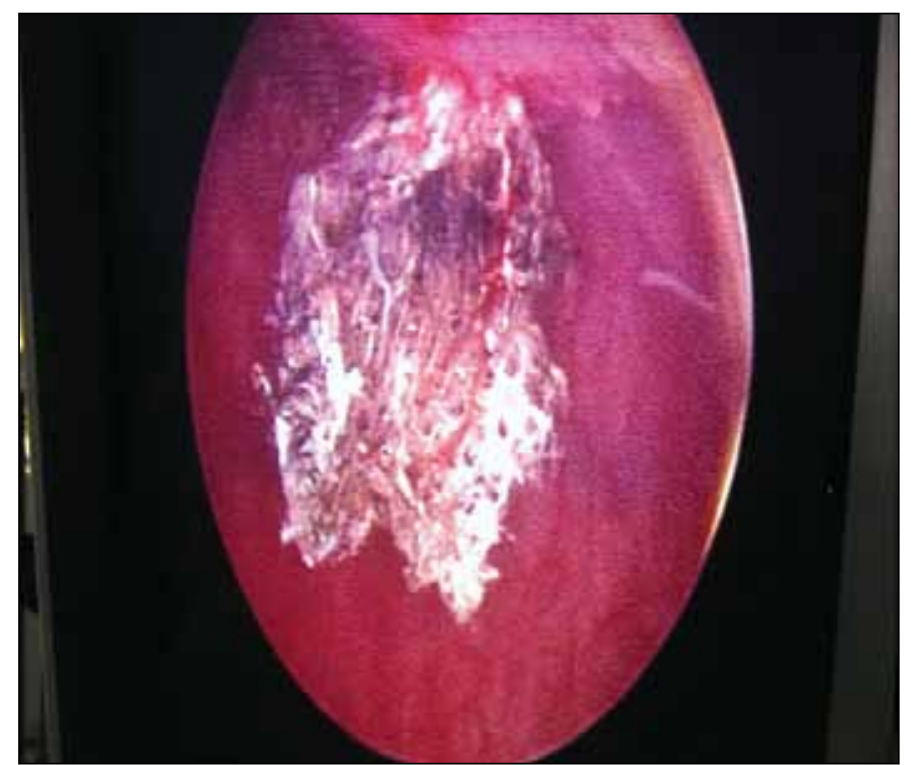

Fig. 5. Diagnostic cystoscopique : image de prothèse de polypropylène faisant saillie dans la vessie.

compliquer la pose du diagnostic ${ }^{21}$ (figure 4).

Ainsi, la radiologie $n^{\prime}$ arrive pas toujours à trancher et souvent une cystoscopie complémentaire est nécessaire. En effet, la cystoscopie reste le moyen le plus fiable pour montrer la protrusion intravésicale de la plaque et la réaction inflammatoire du mur vésical, mais elle reste limitée par la calcification de la plaque et le saignement qui accompagne souvent la cystite (figure 5).

\section{Traitement}

À notre connaissance, aucune étude n'a traité la conduite spécifique à tenir devant une migration de prothèse d'hernie. Théoriquement, le traitement de telles situations dépend du type de migration et des lésions provoquées dont le bilan est apporté par TDM. Une prothèse herniaire déplacée se comporte comme un corps étranger qui agit par érosion et infection des organes avoisinants. L'excision de l'extrémité migrée de la prothèse semble logique dans ce genre de circonstances.

L'exérèse totale de la prothèse n'est pas systématique. En cas de déplacement sans séparation, l'excision de l'extrémité déplacée peut être suffisante. La présence de lésions aux organes avoisinants exige un traitement. Dans le cas d'extrusion intestinale, une résection protégée ou non par stomie selon les conditions locales est une approche classique ${ }^{17}$.

Une réparation sans exérèse semble possible mais hasardeuse. Aucun cas à notre connaissance n'a été rapporté dans la littérature. Le traitement peut être effectué soit par chirurgie ouverte ou laparoscopique. La migration de la prothèse en intravésical offre la possibilité d'un traitement endoscopique (figure 6); le traitement endoscopique ne per-

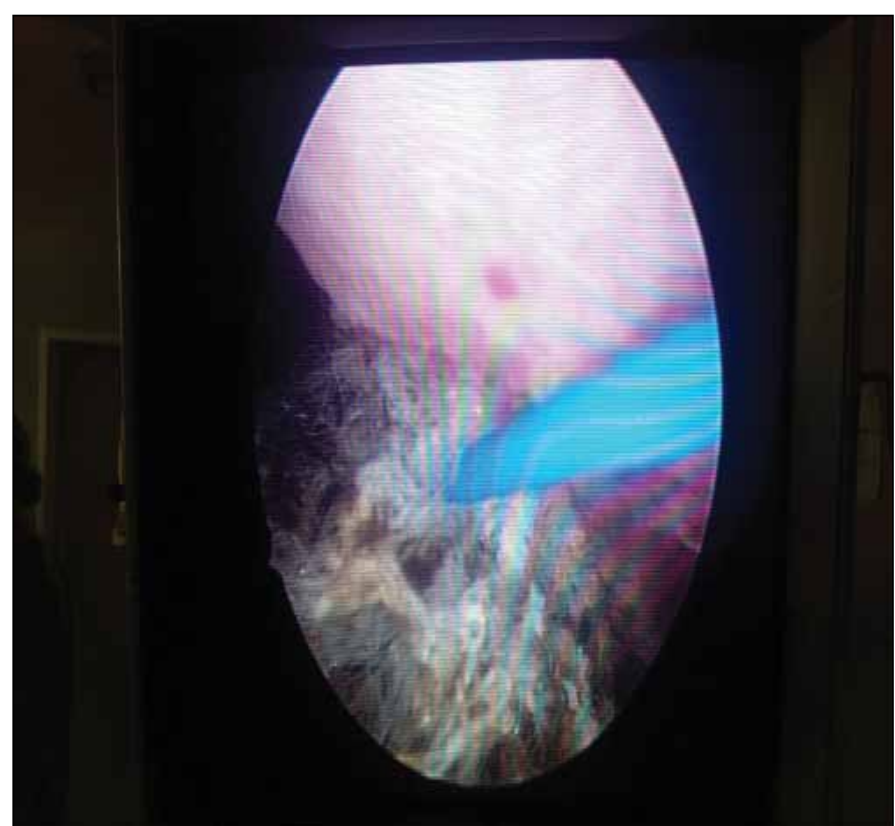

Fig. 6. Césection de la prothèse par voie endoscopique par laser Holmium.

met en général qu'une exérèse partielle de la prothèse, ce qui peut être suffisant en cas de petit orifice de pénétration avec absence d'autres lésions, à condition de garder une sonde vésicale suffisamment longtemps pour permettre une bonne cicatrisation du mur vésical.

Les larges pénétrations obligent un abord chirurgical par chirurgie ouverte sus-pubienne ou laparoscopique, de préférence d'abord transpéritonéal. Une séparation de la prothèse du mur vésical peut s'avérer difficile à cause des adhérences ${ }^{20,22}$.

Des cystectomies partielles peuvent être effectuées de la partie mobile de la vessie. L'existence de foyers infectieux demande un large abord chirurgical permettant l'évacuation des abcès, l'excision des trajets fistuleux, un lavage abondant et un bon drainage postopératoire.

Une antibiothérapie à large spectre, de durée plus ou moins longue, est recommandée. La migration d'une prothèse expose au risque de récidive de I'hernie. Un traitement préventif de l'hernie n'est pas judicieux.

\section{Prévention}

La compréhension des mécanismes physiopathologiques de la migration des plaques synthétiques après hernioplastie permet de dégager quelques mesures de prévention : respect formel des mesures d'asepsie au cours de l'hernioplastie, taillage de la prothèse de manière à émousser les bords aigus, bonne et prudente dissection pour éviter les blessures ou le coinçage des organes avoisinants par les points de suture, bonne fixation de la prothèse aux structures ligamentaires et vérification que la vessie n'est pas trop pleine avant 
de procéder à l'hernioplastie. La lutte contre la surcharge pondérale est un facteur permettant de limiter la surpression abdominale.

\section{Conclusion}

La migration de prothèses synthétiques est un incident rarement rapporté dans la littérature. Si la migration s'effectue en intrapéritonéal, elle peut menacer le pronostic vital. La migration en intravésical est moins grave. Elle pose le problème du diagnostic différentiel devant l'absence de symptômes typiques ou pathognomoniques. L'existence d'une symptomatologie irritative ou d'une hématurie isolée chez un patient avec des antécédents d'hernioplastie doit évoquer le diagnostic de migration endovésicale de la prothèse.

Competing interests: The authors declare no competing financial or personal interests.

This paper has been peer-reviewed.

\section{Références}

1. Riaz AA, Ismail M, Barsam A, et al. Mesh erosion into the bladder: A late complication of incisional hernia repair. A case report and review of the literature. Hernia 2004;8:158-9. http://dx.doi.org/10.1007/ s10029-003-0187-0

2. Hamouda A, Kennedy J, Grant $\mathrm{N}$, et al. Mesh erosion into the urinary bladder following laparoscopic inguinal hernia repair: Is this the tip of the iceberg? Hernia 2010;14:317-9. http://dx.doi.org/10.1007/ s10029-009-0539-5

3. Lichtenstein IL, Shulman AG, Amid PK, et al. The tension-free hernioplasty. Am I Surg 1989;157:188-93. http://dx.doi.org/10.1016/0002-9610(89)90526-6

4. Amid PK. Classification of biomaterials and their related complications in abdominal wall hernia surgery. Hernia 1997;1:15. http://dx.doi.org/10.1007/BF02426382

5. Klinge $U$, Klosterhalfen B. Modified classification of surgical meshes for hernia repair based on the analyses of 1,000 explanted meshes. Hernia 2012;16:251-8. http://dx.doi.org/10.1007/s10029-012-0913-6
6. Iannitti DA, Hope WW, Norton HJ, et al. Technique and outcomes of abdominal incisional hernia repair using a synthetic composite mesh: A report of 455 cases. J Am Coll Surg 2008;206:83-8. http://dx.doi. org/10.1016/i.jamcollsurg.2007.07.030

7. Agrawal A, Avill R. Mesh migration following repair of inguinal hernia: A case report and review of literature. Hernia 2006;10:79-82. http://dx.doi.org/10.1007/s10029-005-0024-8

8. Jacob BP. The SAGES Manual of Hernia Repair. Springer Science Business Media, New York; 2013. http://dx.doi.org/10.1007/978-1-4614-4824-2

9. Earle DB, Mark LA. Prosthetic material in inguinal hernia repair: How do I choose? Surg Clin North Am 2008:88:179-201. http://dx.doi.org/10.1016/i.suc.2007.11.002

10. Binnebosel $M$, Rosch $R$, Junge $K$, et al. Biomechanical analyses of overlap and mesh dislocation in an incisional hernia model in vitro. Surgery 2007;142:365-71. http://dx.doi.org/10.1016/i. surg.2007.04.024

11. Chand $M, O n J$, Bevan K, et al. Mesh erosion following laparoscopic incisional hernia repair. Hernia 2012;16:223.

12. Nowak DD, Chin AC, Singer MA, et al. Large scrotal hernia: A complicated case of mesh migration, ascites, and bowel strangulation. Hernia 2005;9:96-9. http://dx.doi.org/10.1007/s10029-004-0260-3

13. Rettenmaier $S$, Heinemann $H$, Truong JP, et al. Marlex mesh mimicking an adnexal malignancy. Hernia 2009;13:221-3.

14. Barreto $G$, Schoemaker D, Siddins $M$, et al. Colovesical fistula following an open preperitoneal "Kugel" mesh repair of an inguinal hernia. Hernia 2009;13:647-9. http://dx.doi.org/10.1007/s10029-0090496-z

15. Szitkar B, Yzet T. Complications tardives de la chirurgie pariétale abdominale : à propos de trois cas de migration de prothèse dans un organe creux. J Radiol 2010;91:59-64. http://dx.doi.org/10.1016/ S0221-0363(10)70007-X

16. Crespi G, Giannetta E, Mariani F, et al. Imaging of early postoperative complications after polypropylene mesh repair of inguinal hernia. Radiol Med 2004;108:107-15.

17. Celik A, Kutun $S$, Kockar C, et al. Colonoscopic removal of inguinal hernia mesh: Report of a case and literature review. J Laparoendosc Adv Surg Tech A 2005;15:408-10. http://dx.doi.org/10.1089/ lap.2005.15.408

18. Lauwers $P$, Bracke $B$, Hubens $G$, et al. Unusual complications of preperitoneal mesh implantation in the treatment of inguinal hernia. Acta Chir Belg 2003;103:513-6.

19. Jensen $J B$, Jonler $M$, Lund $L$. Recurrent urinary tract infection due to hernia mesh erosion into the bladder. Scand J Urol Nephrol 2004;38:438-9. http://dx.doi.org/10.1080/00365590410031689

20. Rettenmaier MA, Heinemann S, Truong $\mathrm{H}$, et al. Marlex mesh mimicking an adnexal malignancy. Hernia 2009;13:221-3. http://dx.doi.org/10.1007/s10029-008-0426-5

21. Novaretti JPT, Silva RDP, Cotrim CAC, et al. Migration mesh mimicking bladder malignancy after open inguinal hernia repair. Hernia 2012;16:467-70. http://dx.doi.org/10.1007/s10029-010-0760-2

22. $0 j 0 \mathrm{P}$, Abenthroth $\mathrm{A}$, Fiedler $\mathrm{P}$, et al. Migrating mesh mimicking colonic malignancy. Am Surg 2006;72:1210-1.

Correspondence: Dr. Jihad El anzaoui, Department of Urology, Military Hospital Sainte Anne, Toulon, France; iihad.elanzaovi@gmail.com 